\title{
In Situ TEM Evidence of Temperature Dependent Defect Morphology in Heavy Ion Irradiated Nanocrystalline Molybdenum
}

James E. Nathaniel, II ${ }^{1}$, Pranav K. Suri ${ }^{1}$, Jon K. Baldwin ${ }^{2}$, Meimei Li $^{3}$, Marquis A. Kirk ${ }^{3}$, and Mitra L. Taheri ${ }^{1}$

1. Department of Materials Science and Engineering, Drexel University, Philadelphia, Pennsylvania 19104, USA

2. Center for Integrated Nanotechnologies, Los Alamos National Laboratory, Los Alamos, NM 87545, USA

3. Nuclear Engineering Division, Argonne National Laboratory, Lemont, Illinois 60439, USA

The development of materials that can better withstand the operating environment within nuclear reactors is of critical importance for the longevity of existing and the robustness of future nuclear energy systems [1]. It is imperative to explore progressive structural materials and microstructural designs that can be used in new and advanced reactors planned to undergo more extreme environments and longer material lifetime [1]. Of particular interest are nanostructured materials, which contain an extremely high density of interfaces, i.e. grain boundaries [2]. Grain boundaries (GBs) can act as effective sinks for irradiation-induced defects. Individual GB response and the effect of GB density on irradiation-induced defect accumulation are dependent on a number of factors including the GB density and character [3]. Hence, it is important to have a comprehensive knowledge of how specific types of grain boundaries respond to irradiation to develop enhanced radiation resistant materials.

Currently, there is little understanding of the direct link between GB character, GB density, and sink efficiency. Modelers have studied the structural effects of defect absorption at GBs noting how defects are accommodated at GB thereby changing the atomic-structure [4]. This in turn can further affect the GB properties such as sink efficiency and the migration of defects towards GBs. Bai et al. concluded that GBs not only act as defect sinks absorbing interstitials but also as sources reemitting them back to recombine with nearby vacancies [5] suggesting that sink efficiency is a dynamic property. However, little is understood experimentally on how sink efficiency evolves with continued damage and what effect defect mobility has on radiation tolerance. For example, the role of reduced grain size to the nanocrystalline regime and subsequent increase in GB density is not fully understood in the context of dislocation or void behavior near GBs which determines their sink efficiency.

Here we describe a study examining radiation tolerance as a function of grain size, damage fluence, and defect mobility, i.e. irradiation temperature. High-resolution TEM imaging is performed in combination with in situ TEM ion irradiation and automated crystal orientation mapping. We perform in situ TEM ion irradiation in nanocrystalline Mo thin films containing grains ranging in size from $20 \mathrm{~nm}$ to $200 \mathrm{~nm}$ with $1 \mathrm{MeV} \mathrm{Kr}^{2+}$ at 3 different temperatures (room temperature, $400^{\circ} \mathrm{C}$, and $800^{\circ} \mathrm{C}$ ) at IVEM-Tandem facility at Argonne National Laboratory. High-resolution imaging and ACOM is conducted before and after irradiation using a JEOL 2100F equipped with ASTAR NanoMegas. Results from these experiments revealed evidence of temperature sensitivity in defect morphology. Through the analysis of the comparable data sets, it is shown that variance in defect densities resulted due to different defect mobilities. Changes in 
the GB structure as a result of irradiation are also discussed [6].

References:

[1] S.J. Zinkle and G.S. Was, Acta Mater. 61 (2013), 735.

[2] I. J. Beyerlein et al., Prog. Mater. Sci. 74 (2015), 125.

[3] B.P. Uberuaga et al., Sci. Rep. 5 (2015), 9095.

[4] T. Frolov et al., Nat. Commun. 4 (2013), 1899.

[5] X.-M. Bai et al., Science 327 (2010), 1631.

[6] This work was supported by the U.S. Department of Energy, Office of Nuclear Energy under DOE Idaho Operations Office Contract DE-AC07- 051D14517 as part of a Nuclear Science User Facilities experiment.
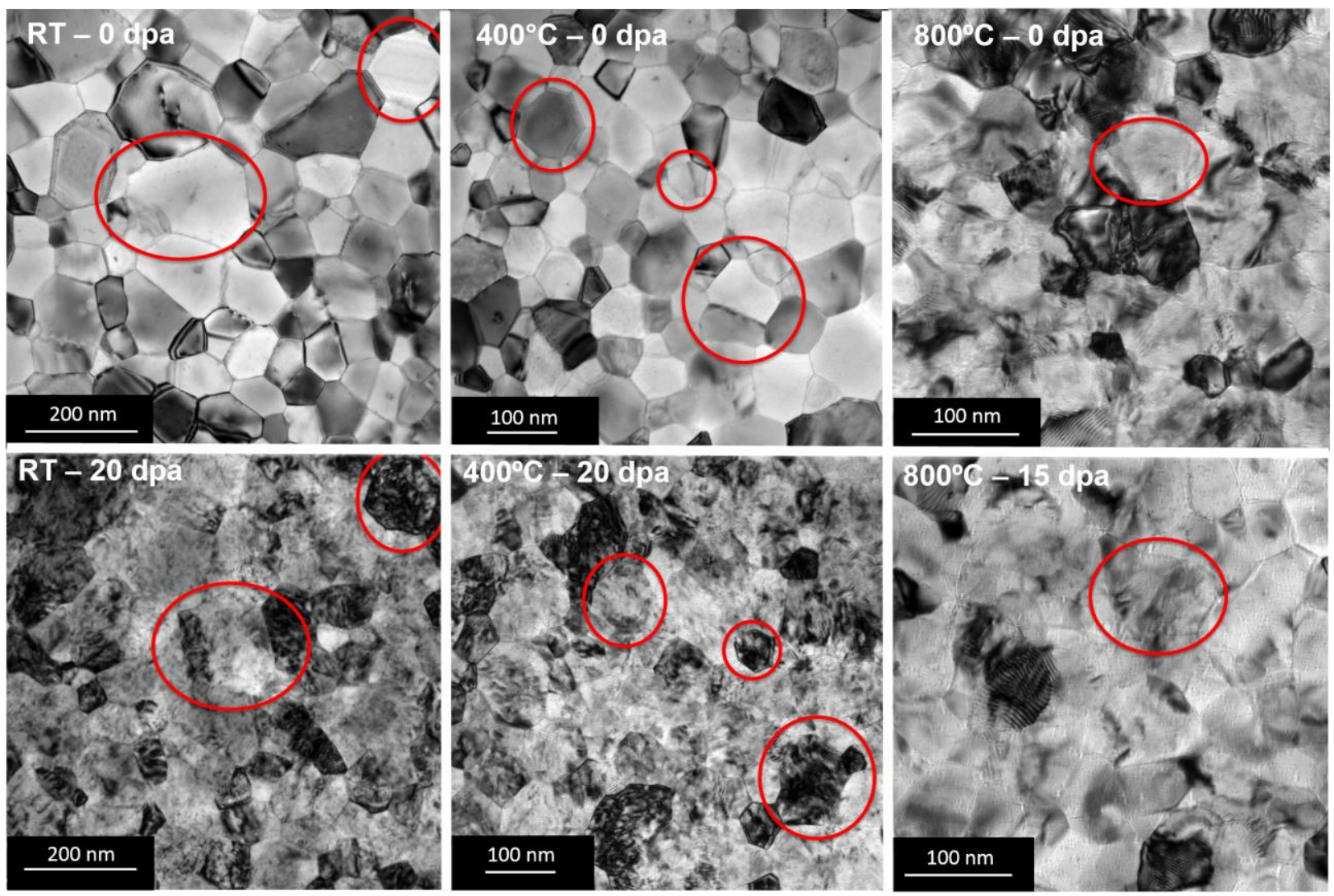

Figure 1. (Left Column) Bright-field TEM micrographs of nanocrystalline Mo films before irradiation via in situ TEM ion irradiation. Irradiation temperature and dose of sample indicated on top left of each micrograph. (Right Column) Bright-field TEM micrographs of nanocrystalline Mo films post irradiation; irradiation temperature and final damage dose indicated on top left of each micrograph. Red circles highlight the same grains pre- and postirradiation in respective samples. 